\title{
Bioanalytical applications in microfluidics
}

\author{
“...applications for microfluidic devices have proliferated at a speed reminiscent of the use of electronics shortly \\ after the invention of the integrated circuit."
}

Keywords: bioanalysis $\approx$ lab-on-a-chip $\approx$ microelectromechanical system $\approx$ microfluidics $\approx$ micro total analysis system

During the past 15 years, one of the greatest research efforts in the field of miniaturization has been in analytical science, with the main goal of this research being to develop miniaturized total analytical systems [1]. This interest in miniaturization has been due to the ever-increasing requirement for utilizing less material, for a variety of reasons. This, in turn, has prompted the design and development of novel miniaturized platforms, hence, the term 'lab-on-a-chip', with corresponding high-throughput methods to accomplish the same tasks as found in larger and older benchtop instruments. For years, the literature has highlighted the 'coming revolution' in microfluidic technology and applications. The term microfluidics is generally used to describe any technology that moves microscopic and nanoscale volumes of fluid through microsized channels on a microelectromechanical system. Microfluidics is the combination of a variety of disciplines including fluid mechanics, surface science, chemistry, physics and biology (and in many cases optics, microscopy, electronics, control systems and microfabrication). The term labon-a-chip is practically passé now for the numbers of papers, patents, inventions and researchers who seek to miniaturize everything larger than a finger. One must now wonder if there is a limit as to what should and can be miniaturized.

Microfluidics has attracted much attention due to the many benefits that miniaturized platforms offer researchers. Advantages include, but are not limited to, small sample volumes leading to greater efficiency of chemical reagents; low production costs per microfluidic device (MD), thereby allowing for disposability; highthroughput synthesis and screening of biological species and drug targets; parallel processing of samples; fast sampling times; accurate and precise control of samples reagents reducing the need for pipetting; low power consumption; and versatile format for integration of various detection schemes thereby leading to greater sensitivity. Fabrication of MDs involves techniques that are well known in the semiconductor industry and manufacturing and have been used in the development of complex devices.

A PubMed query indicates that the citation rate for the word 'microfluidics' increased fivefold between 2001 and 2004 and doubled to 500 between 2003 and 2004. In 1998, the number of issued patents detailing the use of microfluidic platforms in commercial applications was less than 25 ; by 2004 the number had risen to over 350. The microfluidics market for life-science applications reached US\$750 million in 2004 and was expected to reach US $\$ 2$ billion by this year. In 2014, the market of MDs is expected to exceed over US\$3 billion. Drug discovery remains the largest microfluidics market and will continue to grow significantly over the next decade. This is particularly true for the technologies that enable multiplexing. However, the largest growth is expected in the field of point of-care (POC) diagnostics and related technologies. With this being said, there are a myriad of companies that offer MDs for a variety of mostly bioanalytical applications including Agilent, Cellix, Fluidigm, Nanostream and Raindance Technologies.

The past 20 years has seen a huge growth in the number of potential applications of microfluidics focusing on biotechnology, pharmaceuticals, life sciences, defense, public health and agriculture. In the bioanalytical area these applications have included single-cell analysis (cell sorting, cell-based assays and stem cell and neuroscience research) [2], nucleic acid analysis (microarrays and RNA analysis) [3], PCR amplification [4], enzyme reactors and assays [5], gene

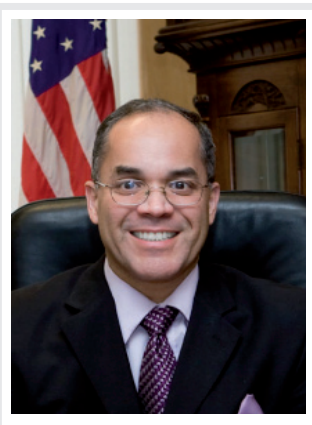

Frank A Gomez

Department of Chemistry \& Biochemistry, California State University, Los Angeles,

5I5I State University Drive, LA, CA 90032-8202, USA Tel.: + I 3233432368 Fax: + I 3233436490 E-mail: fgomez2@calstatela.edu 
isolation and transformation [6], biochemical monitorization [7], in vitro diagnostics (clinical and veterinary diagnostics) [8] and protein crystallization [9]. In fact, applications for MDs have proliferated at a speed reminiscent of the use of electronics shortly after the invention of the integrated circuit.

This special issue on bioanalytical applications in microfluidics brings together the work of scientists and researchers from across several disciplines, to demonstrate the strong overlap between their efforts and how their work typifies the exciting potential of microfluidics in the bioanalytical realm. As can be seen, people with diverse educational backgrounds and training have found microfluidics to be an interesting area of study and it is my hope that this special issue will inspire future scientists to consider studying microfluidics. Only by bringing together people of varied backgrounds can this field reach its full potential.

In this issue, Li et al. detail a study involving transfection of molecular beacons in microfluidic channels. Ueda developed a microfluidics-based noncompetitive immunodetection chip system using anti-T4 antibody fragments obtained from a phage display library. Amasia et al. describe the development and validation of a large-volume centrifugal or CD-based device for blood plasma separation that is capable of processing $2 \mathrm{ml}$ of undiluted blood samples. Lahann highlights the use of reactive coatings for surface modification applications focusing on microfluidic systems. Wang provides an overview of current MDs used in islet studies and analyzes their strengths and experimental suitability. Shadpour reviews the 1D and 2D microchip profiling of proteins for POC applications. Kim reviews the technical aspects and application of image cytometers, recent progress in the field and available commercial devices. The Mora article is a product review of the Gyrolab ${ }^{\circledR}$ microfluidics platform used in ligand binding assays. I hope that readers of this special issue will gain a balanced view of a few of the bioanalytical applications in microfluidics, and entertain the thought of integrating them into their research program.

\section{Financial \& competing interests disclosure}

The author gratefully acknowledges financial support for this research by grants from the National Science Foundation (CHE-0515363, DMR-0351848 and CBET0723271). The author has no other relevant affiliations or financial involvement with any organization or entity with a financial interest in or financial conflict with the subject matter or materials discussed in the manuscript apart from those disclosed.

No writing assistance was utilized in the production of this manuscript.

\section{Bibliography}

1 West J, Becker M, Tombrink S, Manz A. Micro total analysis systems. Latest achievements. Anal Chem. 80, 4403-4419 (2008).

2 Mellors JS, Jorabchi K, Smith LM, Ramsey JM. Integrated microfluidic device for automated single cell analysis using electrophoretic separation and electrospray ionization mass spectrometry. Anal Chem. 82, 967-973 (2010).

3 Hopwood AJ, Hurth C, Yang J et al. Integrated microfluidic system for rapid forensic DNA analysis: sample collection to DNA profile. Anal Chem. 82, 6991-6999 (2010).
4 Shaw KJ, Docker PT, Yelland JV et al. Rapid PCR amplification using a microfluidic device with integrated microwave heating and air impingement cooling. Lab Chip 10, 1725-1728 (2010).

5 Liu X, Lo RC, Gomez FA. Fabrication of a microfluidic enzyme reactor utilizing magnetic beads. Electrophoresis 30, 2129-2133 (2009).

6 Toriello NM, Douglas ES, Thaltrong N et al. Integrated microfluidic bioprocessor for single-cell gene expression analysis. Proc. Natl Acad. Sci. USA 105, 20173-20178 (2008).
7 Roman GT, Kennedy RT. Fully integrated microfluidic separations systems for biochemical analysis. J. Chromatogr. A 1168, 170-188 (2007).

8 Martinez AW, Phillips ST, Whitesides GM, Carrilho E. Diagnostics for the developing world: microfluidic paper-based analytical devices. Anal. Chem. 82, 3-10 (2010).

9 Li L, Ismagilov RF. Protein crystallization using microfluidic technologies based on valves, droplets and slipchip. Annu. Rev. Biophysics. 39, 139-158 (2010). 